\title{
In vitro study of the antifungal potential of Glycyrrhiza glabra L. against Candida species
}

N Martins $\stackrel{1}{1} \underline{2}$, S Sónia $\stackrel{1}{-}$, L Barros $\stackrel{2}{-}$ I Ferreira $\stackrel{2}{2}$, M Henriques $\stackrel{1}{-}$

- ${ }^{1}$ IBB - Institute for Biotechnology and Bioengineering, Centre of Biological Engineering, University of Minho, Braga, Portugal

- ${ }^{2}$ Mountain Research Centre (CIMO), ESA, Polytechnic Institute of Bragança, Portugal

- $\underline{\text { Congress Abstract }}$

In the last two decades, an increasing incidence of opportunistic fungal infections has been observed, not only at hospital level but also in the community. Candida albicans was identified as the main responsible agent for those infections, but other non-Candida albicans Candida (NCAC) species have also been associated with this complicated medical condition [1]. Furthermore, higher rates of fungal resistance to the current drugs have been observed and are still growing, making the discovery of new active antifungal substances very urgent. Medicinal plants can be explored for that purpose. Glycyrrhiza glabra L. is commonly used in folk medicine due to its antimicrobial and antiviral activities, as well as for a wide variety of dermal affections [2]. Some reports have described the antimicrobial potential of licorice (G. glabra roots), namely aqueous extracts [e.g., 3]. Herein, the antifungal potential of the hydroalcoholic extract prepared from rhizomes and roots of G. glabra, was evaluated against 19 Candida strains, using the disc diffusion halo assay. The licorice extract was effective against all the tested C. albicans, $C$. glabrata, $C$. parapsilosis and $C$. tropicalis strains. It is very interesting to highlight the broad spectrum of activity of this extract, for which were observed similar results into different strains of the same species and even between different species. The results for the inhibitory zones, at the tested concentration $(50 \mathrm{mg} / \mathrm{mL})$, after $24 \mathrm{~h}$, were $1.0-1.2 \mathrm{~cm}$ for C. albicans and C. parapsilosis, $1.0-1.3 \mathrm{~cm}$ for C. tropicalis and $1.2 \mathrm{~cm}$ for C. glabrata, maintaining the potential at $48 \mathrm{~h}$ and even at $72 \mathrm{~h}$. This study demonstrates that G. glabra hydroalcoholic extract probably exerts mainly fungicidal and not so much fungistatic effects, and it could find applications in antifungal therapy. Nevertheless, further studies will be performed in order to characterize the extract, namely on phenolic composition, and to elucidate its mechanism of action.

Keywords: Antifungal activity; Candida species; Medicinal plants; Glycyrrhiza glabra L.;

References:

[1] Martins N, Ferreira ICFR, Barros L, Silva S, Henriques M. (2014). Mycopathol. 177, 223 240.

[2] Asl MN, Hosseinzadeh H. (2008). Phyther Res. 22, 709 - 24.

[3] Irani M, Sarmadi M, Bernard F, Ebrahimi GH, Bazarnov HS. (2010). Iran J Pharm Res. 9, 425 -8 . 